Review Article

\title{
Obesity as a Possible Risk Factor for Progression from Monoclonal Gammopathy of Undetermined Significance Progression into Multiple Myeloma: Could Myeloma Be Prevented with Metformin Treatment?
}

\author{
Ademar Dantas da Cunha Júnior $\mathbb{D}^{1,2,3}$ Dalila Luciola Zanette $\mathbb{D}^{4},{ }^{4}$ \\ Fernando Vieira Pericole $\mathbb{D}^{5},{ }^{5}$ Sara Teresinha Olalla Saad $\left(\mathbb{D},{ }^{5}\right.$ \\ and José Barreto Campello Carvalheira (D) $^{1}$ \\ ${ }^{1}$ Division of Oncology, Department of Internal Medicine, Faculty of Medical Sciences, State University of Campinas (UNICAMP), \\ Campinas, SP, Brazil \\ ${ }^{2}$ Hematology and Oncology Clinics, Cancer Hospital of Cascavel, União Oeste de Estudos e Combate ao Câncer (UOPECCAN), \\ Cascavel, PR, Brazil \\ ${ }^{3}$ Department of Internal Medicine, State University of Western Paraná (UNIOESTE), Cascavel, PR, Brazil \\ ${ }^{4}$ Laboratory for Applied Science and Technology in Health, Carlos Chagas Institute (ICC), Oswaldo Cruz Foundation (Fiocruz), \\ Rio de Janeiro, Brazil \\ ${ }^{5}$ Hematology and Blood Transfusion Center, University of Campinas (UNICAMP), Campinas, SP, Brazil
}

Correspondence should be addressed to Ademar Dantas da Cunha Júnior; ademardcj@gmail.com

Received 5 December 2020; Revised 22 December 2020; Accepted 8 January 2021; Published 18 January 2021

Academic Editor: David H. Vesole

Copyright (c) 2021 Ademar Dantas da Cunha Júnior et al. This is an open access article distributed under the Creative Commons Attribution License, which permits unrestricted use, distribution, and reproduction in any medium, provided the original work is properly cited.

\begin{abstract}
Obesity is increasingly associated with the transformation of monoclonal gammopathy of undetermined significance (MGUS) into multiple myeloma (MM). Obesity, MGUS, and MM share common etiopathogenesis mechanisms including altered insulin axis and the action of inflammatory cytokines. Consistent with this interconnection, metformin could predominantly exert inhibition of these pathophysiological factors and thus be an attractive therapeutic option for MGUS. Despite the possible clinical significance, only a limited number of epidemiological studies have focused on obesity as a risk factor for MGUS and MM. This review describes multiple biological pathways modulated by metformin at the cellular level and their possible impacts on the biology of MGUS and its progression into MM.
\end{abstract}

\section{Introduction}

Brazilian studies have revealed a remarkable prevalence of diabetes [1], obesity [2], and cancer [3] in adults over time and with a progressive expansion in the last years. Diabetes mellitus type 2 (DM2) and cancer share many risk factors, especially obesity and metabolic syndrome, with potential biological connections between the two diseases, as metaanalysis data from prospective cohort studies suggest a modest but consistent direct effect of body mass index (BMI) on the incidence of lymphoma, multiple myeloma, increased risk of leukemia in adults [4-13], increased risk of monoclonal gammopathy of undetermined significance (MGUS) transformation into $\mathrm{MM}[5,12,14]$, and increased risk of death in MM patients [15]. Data linking obesity to myeloma are the most convincing among hematologic malignancies, based on their replication in most studies [16-18]. Although metformin has been shown to modulate multiple biological pathways at the cellular level [19], few studies have focused on the effects of metformin on the biology of MGUS and the 
protection from MM transformation. Although MM therapeutic approaches conferred remarkable progress in the last decade, the mortality of this disease is still considerably high. Moreover, the therapeutic approaches available following relapse are not feasible in low-income countries and are financially costly for the public health system $[20,21]$. Therefore, low-cost drugs that have a positive impact on prevention should be carefully studied as an attempt to avoid MGUS into MM progression.

\section{Discussion}

2.1. Obesity in MM. Three percent of the population above 50 and 5\% above 70 years of age has MGUS [22]. The incidence of progression into myeloma, lymphoma, or amyloidosis among patients with MGUS is about $1 \%$ per year [22]. MGUS and MM share common risk factors that include occupational exposure to benzene and pesticides $[23,24]$, advanced age [25], African ancestry [26, 27], and male gender [25]. Recent evidence suggests common genetic susceptibility to MM and MGUS [28-30] but still require further studies. It should be noted that, in recent years, there is growing evidence that obesity acts as a risk factor for the occurrence of MM [4, 16-18, 31, 32]. In addition, obesity affects the transformation of MGUS into MM [12, 33, 34]. From the epidemiological point of view, there are prospective as well as meta-analyses data that support the association between increased BMI and increased risk of developing hematological malignancies [4, 9, 15, 35-37]. However, the role of confounder factors for this association such as dietary habits, physical activity, and/or antidiabetic therapy is largely unknown [38].

The modified insulin signaling axis [32-35], the release of adipokines [36-41], and lastly, low-grade chronic inflammation $[34,42]$ are plausible molecular mechanisms for the association between obesity and hematological neoplasms. Leptin in combination with bone marrow microenvironment cytokines, for example, interleukine-6 (IL-6), insulin-like growth factor-1 (IGF-1), vascular endothelial growth factor (VEGF), and TNF-alpha lead to adipocyte differentiation of bone marrow mesenchymal stromal cells (BM-MSCs), thus contributing to an increase in the adipose marrow tissue $[39,40]$. Therefore, all these mediators negatively affect hematopoiesis and subsequently lead to the remodeling of the bone marrow microenvironment that is observed in obesity which favors the growth of cells neoplasms in bone marrow (BM) [37-39]. Likewise, IGF-1 and IL-6 receptors show JAK-independent synergistic effects on the induction of MM cell growth [41]. IGF-1 is an endocrine factor produced and secreted by bone marrow stromal cells (BMSC), bone endothelial cells, and osteoblasts that promotes the homing, growth, and survival of myeloma cells in the bone marrow environment, both dependent and independent of IL-6 [42]. The binding of the ligand to the IGF-1 receptor (IGF-1R) via the tyrosine kinase (TK) domain activates the phosphoinositide 3-kinases/protein kinase B (PI3K/Akt) $[43,44]$ and mitogen-activated protein/extracellular signal-regulated kinases (MAPK/MEK/ERK) signaling pathways [43, 44]. Indeed, IGF-1 mediates multiple effects on
MM cells through different signal transduction pathways. IGF1 binding to IGF-1R triggers tyrosine kinase, resulting in activation of PI3K/Akt and RAS/RAF/MEK-ERK. PI3K/Akt is involved mainly in the antiapoptotic effects, whereas the MEK-ERK regulates cell cycle and proliferation. In addition to these main pathways, IGF-IR is able to activate the Janus kinases/signal transducer and activator of transcription proteins (JAK/STAT) pathway and Wnt and nuclear factor kappa-light-chain-enhancer of activated B cells (NF- $\kappa \mathrm{B})$ signaling [45] IGF-1 induces VEGF production in MM cells through the MEK/ERK pathway, which increases angiogenesis in the BM [46, 47]. IL-6 is a cytokine with pleiotropic effects in hematopoietic and nonhematopoietic cells [39] with prognostic value in MM $[48,49]$. IL-6 is mostly secreted by the BMSC, and its production and secretion are enhanced by the adhesion between MM cells and BMSC, via NFkB [39]. VEGF and IL- 6 show mutual stimulation in MM, in which VEGF acts as a paracrine mediator that supports MM cell growth through the increase of IL- 6 secretion by BMSCs and microvascular endothelial cells. IL-6, in turn, may stimulate VEGF secretion in a subset of myeloma cells, indirectly promoting angiogenesis $[50,51]$. Following binding to its receptor, IL- 6 is able to trigger the activation of the MEK/MAPK [52], JAK/STAT [53], and PI3K/AKT signaling pathways [54].

Another essential signaling pathway that correlates with the development of myeloma bone disease (MBD) is the receptor activator of nuclear factor $\kappa \mathrm{B}$ (RANK)/ligand of RANK (RANKL) pathway, which stimulates the signals via NF- $\kappa \mathrm{B}$ and MAPK pathway providing the development, maturation, and differentiation of osteoclast precursors [55]. These factors stimulate cell surface receptors and signaling through JAK/STATs, MAPK, and PI3K molecules frequently dysregulated in neoplastic cells [56].

Obesity and myeloma share signaling pathways that upregulate insulin, IGF-1, leptin, and inflammatory cytokines, raising the risk of malignant transformation $[57,58]$. Consonant with this, a drug that potentially inhibits these pathways could be interesting as a therapeutic alternative in MGUS consequently being associated with a lower risk to develop MM [34]. The malignant transformation of a post-GC B cell or plasma cell into MGUS and consequently into MM requires both an initiating genetic event and multiple secondary genetic events $[59,60]$. The malignant evolution of MGUS is mediated by structural and functional alterations of the tumor-associated stromal cells, producing in BM microenvironment that acts as an essential partner in carcinogenesis and hence can be a new target for therapy in early disease stages [59].

2.2. Metformin as a Preventive Drug in MM. The Diabetes Prevention Program (DPP) study showed that metformin constantly reduced body weight over time, which could explain the diabetes prevention effects of the drug. Metformin also significantly improved fasting insulin and proinsulin and other adiposity parameters such as BMI, waist circumference, and waist-hip ratio [61].

Epidemiological and preclinical research studies indicate that metformin is a potential therapeutic target in patients with leukemia [62, 63], lymphomas [63-65], and multiple myeloma 


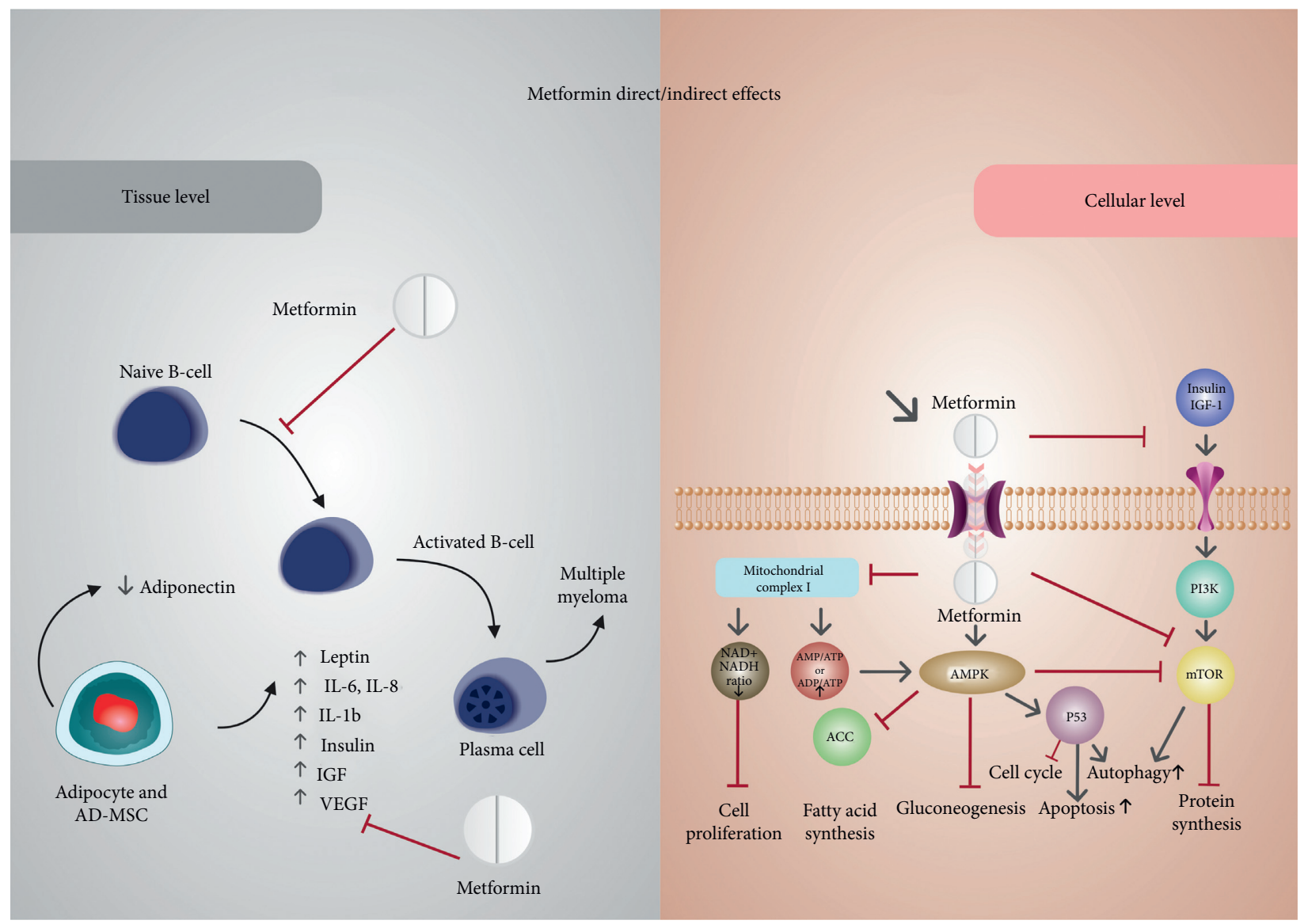

Figure 1: Figure modified from [32]. Overview of cellular and tissue mechanisms of metformin on inhibiting plasm cell growth. Metformin inhibits at tissue level: the inflammation caused by increases in circulating levels of leptin, insulin, IGF, IL-6, IL-1, and VGFR, driving by adipocyte differentiation of bone marrow mesenchymal stromal cells (AD-MSCs); and a cellular level inhibits mitochondria complex I, stimulates the adenosine monophosphate-activated protein kinase (AMPK) signaling pathway, and/or inhibits the insulin signaling pathway. ACC, acetyl-CoA carboxylase; EMT, epithelial-mesenchymal transition; IGF, insulin growth factor; IGF-1, insulin-like growth factor-1; IGF-1R, insulin-like growth factor-1 receptor; IR, insulin receptor; IL-1, interleukin 1; IL-6, interleukin-6, OCT1, organic cation transporter 1; VGFR, endothelial growth factor.

$[33,34,66-68]$. It is expected that the diverse pleiotropic effects of the drug act on multiple targets, specifically in myeloma, in which there is a strong interaction between the clonal plasma cells and the BM microenvironment [69] (Figure 1).

Metformin might conduct to systemically reduce the levels of proinflammatory soluble mediators (e.g., IL-6 and IGF-1) [70]. A recent study showed metformin specifically decreased IL-6R expression, which is mediated via AMPK, mTOR, as well as displaying an isolated and synergistic effect with common antimyeloma drugs. Intriguingly, IL$6 \mathrm{R}$ can serve as a biomarker for metformin action in multiple myeloma [70]. Besides, it promotes appropriate direction and intensity, antitumor immunity-related metabolic checkpoints not solely in T cells, cancer cells, and associated immune suppressor cells of the microenvironment; furthermore, it might interfere with the gut microbiota and its systemic impacts on body metabolism [71].

Finally, metformin influences bone turnover because it activates AMP-activated protein kinase (AMPK), which, in turn, acts as a negative regulator of RANKL in the differentiation of osteoclasts. Furthermore, metformin might also significantly suppress bone resorption [72, 73].

New epidemiological and preclinical research points to metformin as a potential therapeutic target in patients with multiple myeloma $[5,12,33,34,68]$. Although epidemiological studies have reasonably and consistently reported reduced MM incidence and/or mortality in diabetic patients who receive metformin [74-78], many sampled their case study retrospectively from the hospital or clinical registries rather than population-based registries, restricting external validity and inserting potential selection biases. Some studies did not exclude individuals with a prior cancer diagnosis, thus presenting a possible reverse causation bias. Many studies included patients exposed to various treatments for diabetes, complicating the analysis of metformin associations. Self-reporting of crucial data such as concomitant medication use and cancer risk factors such as obesity, tobacco use, and family history may have provided exposure biases. Moreover, research of metformin benefit markers will demand to include essential host factors such as circulating insulin and glucose levels, obesity, and expression of OCT1 receptors in the liver and the tumor 
[79]. Additionally, tumor cells' characteristics such as receptors (insulin/IGF1) [57, 80] and pathway proteins (PI3K/mTOR, LKB1, and TSC2) [81, 82] expression might potentially mediate these indirect, host-mediated effects and any direct effects that are extremely important.

Currently, there are a reduced number of epidemiological studies focused on obesity as a risk factor for MGUS and $\mathrm{MM}$, notwithstanding the potential clinical importance of obesity in myeloma [83]. Obesity and DM2 are risk factors for myeloma that make metformin a potential protective therapy in the evolution of the natural history of the disease $[12,34]$.

\section{Conclusion}

Assuming that $3 \%$ of adults over the age of 50 have MGUS and the increasing numbers of diabetes and obesity in the world population, less toxic approaches are needed to minimize the chances of MGUS to MM progression. Antidiabetic drugs such as metformin are low-cost and safe, and studies have demonstrated their potentially protective roles in cancer, MM, and MGUS to MM progression. Interventions with minor results may have a meaningful influence on the cumulative disease load. Considering the pleiotropic effects (direct and indirect) of metformin on the bone marrow milieu, it is essential to investigate the mechanisms involved in the preventive effects of the drug in the progression of multiple myeloma, in order to indicate its proper use in this context.

\section{Data Availability}

The data used to support the findings of this study are available from the corresponding author upon request.

\section{Conflicts of Interest}

The authors declare that they have no conflicts of interest.

\section{Authors' Contributions}

ADCJ, DLZ, and JBCC designed the review; ADCJ and DLZ wrote the manuscript. FVP, STOS, and JBCC reviewed the manuscript and provided comments. All authors reviewed and approved the final manuscript. Ademar Dantas da Cunha Júnior and Dalila Luciola Zanette contributed equally to this work.

\section{Acknowledgments}

The authors thank all their colleagues who provided insight and expertise that greatly assisted in the review. The authors also acknowledge Wagner Nagib de Souza Birbeire for his comprehension ability to make the picture drawing according to their rational.

\section{References}

[1] G. H. Telo, F. V. Cureau, M. S. de Souza, T. S. Andrade, F. Copes, and B. D. Schaan, "Prevalence of diabetes in Brazil over time: a systematic review with meta-analysis," Diabetology \& Metabolic Syndrome, vol. 8, no. 1, p. 65, 2016.

[2] D. C. K. Gomes, R. Sichieri, E. V. Junior, C. S. Boccolini, A. de Moura Souza, and D. B. Cunha, "Trends in obesity prevalence among Brazilian adults from 2002 to 2013 by educational level," BMC Public Health, vol. 19, no. 1, p. 965, 2019.

[3] I. R. Barbosa, D. L. B. D. Souza, M. M. Bernal, and Í. D. C. C. Costa, "Cancer mortality in Brazil," Medicine, vol. 94, no. 16, p. e746, 2015.

[4] A. G. Renehan, M. Tyson, M. Egger, R. F. Heller, and M. Zwahlen, "Body-mass index and incidence of cancer: a systematic review and meta-analysis of prospective observational studies," The Lancet, vol. 371, no. 9612, pp. 569-578, 2008.

[5] O. Landgren, S. V. Rajkumar, R. M. Pfeiffer et al., "Obesity is associated with an increased risk of monoclonal gammopathy of undetermined significance among black and white women," Blood, vol. 116, no. 7, pp. 1056-1059, 2010.

[6] S. C. Larsson and A. Wolk, "Body mass index and risk of nonHodgkin's and Hodgkin's lymphoma: a meta-analysis of prospective studies," European Journal of Cancer, vol. 47, no. 16, pp. 2422-2430, 2011.

[7] P. Fernberg, Å. Odenbro, R. Bellocco et al., "Tobacco use, body mass index, and the risk of leukemia and multiple myeloma: a nationwide cohort study in Sweden," Cancer Research, vol. 67, no. 12, pp. 5983-5986, 2007.

[8] S. C. Larsson and A. Wolk, "Body mass index and risk of multiple myeloma: a meta-analysis," International Journal of Cancer, vol. 121, no. 11, pp. 2512-2516, 2007.

[9] S. C. Larsson and A. Wolk, "Overweight and obesity and incidence of leukemia: a meta-analysis of cohort studies," International Journal of Cancer, vol. 122, no. 6, pp. 1418-1421, 2008.

[10] A. Wallin and S. C. Larsson, "Body mass index and risk of multiple myeloma: a meta-analysis of prospective studies," European Journal of Cancer, vol. 47, no. 11, pp. 1606-1615, 2011.

[11] J. J. Castillo, F. Mulkey, S. Geyer et al., "Relationship between obesity and clinical outcome in adults with acute myeloid leukemia: a pooled analysis from four CALGB (alliance) clinical trials," American Journal of Hematology, vol. 91, no. 2, pp. 199-204, 2016.

[12] S. H. Chang, S. Luo, T. S. Thomas et al., "Obesity and the transformation of monoclonal gammopathy of undetermined significance to multiple myeloma: a population-based cohort study," Journal of the National Cancer Institute, vol. 109, no. 5, 2017.

[13] Q. J. N. Leo, N. J. Ollberding, L. R. Wilkens et al., “Obesity and non-Hodgkin lymphoma survival in an ethnically diverse population: the multiethnic cohort study," Cancer Causes \& Control, vol. 25, no. 11, pp. 1449-1459, 2014.

[14] M. Thordardottir, E. K. Lindqvist, S. H. Lund et al., "Obesity and risk of monoclonal gammopathy of undetermined significance and progression to multiple myeloma: a populationbased study," Blood Advances, vol. 1, no. 24, pp. 2186-2192, 2017.

[15] E. E. Calle, C. Rodriguez, K. Walker-Thurmond, and M. J. Thun, "Overweight, obesity, and mortality from cancer in a prospectively studied cohort of U.S. adults," New England Journal of Medicine, vol. 348, no. 17, pp. 1625-1638, 2003. 
[16] M. A. Lichtman, "Obesity and the risk for a hematological malignancy: leukemia, lymphoma, or myeloma," The Oncologist, vol. 15, no. 10, pp. 1083-1101, 2010.

[17] B. Lauby-Secretan, C. Scoccianti, D. Loomis et al., "Body fatness and cancer-viewpoint of the IARC working group," New England Journal of Medicine, vol. 375, no. 8, pp. 794-798, 2016.

[18] M. Kyrgiou, I. Kalliala, G. Markozannes et al., "Adiposity and cancer at major anatomical sites: umbrella review of the literature," BMJ, vol. 356, p. j477, 2017.

[19] A. Cunha Júnior, F. Pericole, and J. Carvalheira, "Metformin and blood cancers," Clinics, vol. 73, no. 1, p. e412s, 2018.

[20] V. T. M. Hungria, A. Maiolino, A. Maiolino et al., "Observational study of multiple myeloma in Latin America," Annals of Hematology, vol. 96, no. 1, pp. 65-72, 2017.

[21] V. T. M. Hungria, J. H. Lee, A. Maiolino et al., "Survival differences in multiple myeloma in Latin America and Asia: a comparison involving 3664 patients from regional registries," Annals of Hematology, vol. 98, no. 4, pp. 941-949, 2019.

[22] R. A. Kyle, T. M. Therneau, S. V. Rajkumar et al., "A long-term study of prognosis in monoclonal gammopathy of undetermined significance," New England Journal of Medicine, vol. 346, no. 8, pp. 564-569, 2002.

[23] D. E. Bergsagel, O. Wong, P. L. Bergsagel et al., "Benzene and multiple myeloma: appraisal of the scientific evidence," Blood, vol. 94, no. 4, pp. 1174-1182, 1999.

[24] C. Perrotta, A. Staines, M. Codd et al., "Multiple myeloma and lifetime occupation: results from the EPILYMPH study," Journal of Occupational Medicine and Toxicology, vol. 7, no. 1, p. 25, 2012.

[25] C. T' Mannetje, "Aetiology: the path to disease," Nature, vol. 480, no. 7377, pp. S54-S55, 2011.

[26] G. D. Smith, J. D. Neaton, D. Wentworth, R. Stamler, and J. Stamler, "Mortality differences between black and white men in the USA: contribution of income and other risk factors among men screened for the MRFIT," The Lancet, vol. 351, no. 9107, pp. 934-939, 1998.

[27] O. Landgren, G. Gridley, I. Turesson et al., "Risk of monoclonal gammopathy of undetermined significance (MGUS) and subsequent multiple myeloma among African American and white veterans in the United States," Blood, vol. 107, no. 3, pp. 904-906, 2006.

[28] A. I. Clay-Gilmour, M. A. T. Hildebrandt, E. E. Brown et al., "Coinherited genetics of multiple myeloma and its precursor, monoclonal gammopathy of undetermined significance," Blood Advances, vol. 4, no. 12, pp. 2789-2797, 2020.

[29] H. Thomsen, S. Chattopadhyay, N. Weinhold et al., "Genome-wide association study of monoclonal gammopathy of unknown significance (MGUS): comparison with multiple myeloma," Leukemia, vol. 33, no. 7, pp. 1817-1821, 2019.

[30] S. Chattopadhyay, H. Thomsen, N. Weinhold et al., "Eight novel loci implicate shared genetic etiology in multiple myeloma, AL amyloidosis, and monoclonal gammopathy of unknown significance," Leukemia, vol. 34, no. 4, pp. 1187-1191, 2020.

[31] B. M. Birmann, G. Andreotti, A. J. De Roos et al., "Young adult and usual adult body mass index and multiple myeloma risk: a pooled analysis in the international multiple myeloma consortium (IMMC)," Cancer Epidemiology Biomarkers \& Prevention, vol. 26, no. 6, pp. 876-885, 2017.

[32] K. R. Carson, M. L. Bates, and M. H. Tomasson, "The skinny on obesity and plasma cell myeloma: a review of the literature," Bone Marrow Transplantation, vol. 49, no. 8, pp. 1009-1015, 2014.

[33] S.-H. Chang, S. Luo, K. K. O’Brian et al., "Association between metformin use and progression of monoclonal gammopathy of undetermined significance to multiple myeloma in US veterans with diabetes mellitus: a population-based retrospective cohort study," The Lancet Haematology, vol. 2, no. 1, pp. e30-e36, 2015.

[34] B. Boursi, R. Mamtani, Y. X. Yang, and B. M. Weiss, "Impact of metformin on the progression of MGUS to multiple myeloma," Leukemia \& Lymphoma, vol. 58, no. 5, pp. 1265-1267, 2016.

[35] E. J. Gallagher and D. LeRoith, "Diabetes, antihyperglycemic medications and cancer risk," Current Opinion in Endocrinology \& Diabetes and Obesity, vol. 20, no. 5, pp. 485-494, 2013.

[36] S. C. Larsson and A. Wolk, "Obesity and risk of nonHodgkin's lymphoma: a meta-analysis," International Journal of Cancer, vol. 121, no. 7, pp. 1564-1570, 2007.

[37] H. Ulmer, T. Bjørge, H. Concin et al., "Metabolic risk factors and cervical cancer in the metabolic syndrome and cancer project (Me-Can)," Gynecologic Oncology, vol. 125, no. 2, pp. 330-335, 2012.

[38] J. J. Castillo, N. Mull, J. L. Reagan, S. Nemr, and J. Mitri, "Increased incidence of non-Hodgkin lymphoma, leukemia, and myeloma in patients with diabetes mellitus type 2: a metaanalysis of observational studies," Blood, vol. 119, no. 21, pp. 4845-4850, 2012.

[39] M. Lemaire, S. Deleu, E. De Bruyne, E. Van Valckenborgh, E. Menu, and K. Vanderkerken, "The microenvironment and molecular biology of the multiple myeloma tumor," Advances in Cancer Research, vol. 110, pp. 19-42, 2011.

[40] E. V. Morris and C. M. Edwards, "Adipokines, adiposity, and bone marrow adipocytes: dangerous accomplices in multiple myeloma," Journal of Cellular Physiology, vol. 233, no. 12, pp. 9159-9166, 2018.

[41] S. Abroun, H. Ishikawa, N. Tsuyama et al., "Receptor synergy of interleukin-6 (IL-6) and insulin-like growth factor-I in myeloma cells that highly express IL-6 receptor $\alpha$," Blood, vol. 103, no. 6, pp. 2291-2298, 2004.

[42] M. Ferlin, N. Noraz, C. Hertogh, J. Brochier, N. Taylor, and B. Klein, "Insulin-like growth factor induces the survival and proliferation of myeloma cells through an interleukin-6-independent transduction pathway," British Journal of Haematology, vol. 111, no. 2, pp. 626-634, 2000.

[43] G. G. Freund, D. T. Kulas, and R. A. Mooney, "Insulin and IGF-1 increase mitogenesis and glucose metabolism in the multiple myeloma cell line, RPMI 8226," Journal of Immunology (Baltimore, Md.: 1950), vol. 151, no. 4, pp. 1811-1820, 1993.

[44] K. Vanderkerken, K. Asosingh, F. Braet, I. Van Riet, and B. Van Camp, "Insulin-like growth factor-1 acts as a chemoattractant factor for 5T2 multiple myeloma cells," Blood, vol. 93, no. 1, pp. 235-241, 1999.

[45] L. Bieghs, H. E. Johnsen, K. Maes et al., "The insulin-like growth factor system in multiple myeloma: diagnostic and therapeutic potential," Oncotarget, vol. 7, no. 30, pp. 48732-48752, 2016.

[46] E. Menu, R. Kooijman, E. V. Valckenborgh et al., "Specific roles for the PI3K and the MEK-ERK pathway in IGF-1stimulated chemotaxis, VEGF secretion and proliferation of multiple myeloma cells: study in the 5T33MM model," British Journal of Cancer, vol. 90, no. 5, pp. 1076-1083, 2004. 
[47] P. Gual, V. Baron, V. Lequoy, and E. Van Obberghen, "Interaction of Janus kinases JAK-1 and JAK-2 with the insulin receptor and the insulin-like growth factor-1 Receptor1," Endocrinology, vol. 139, no. 3, pp. 884-893, 1998.

[48] D. M. Nachbaur, M. Herold, A. Maneschg, and H. Huber, "Serum levels of interleukin-6 in multiple myeloma and other hematological disorders: correlation with disease activity and other prognostic parameters," Annals of Hematology, vol. 62, no. 2-3, pp. 54-58, 1991.

[49] V. M. Lauta, "A review of the cytokine network in multiple myeloma," Cancer, vol. 97, no. 10, pp. 2440-2452, 2003.

[50] T. Cohen, D. Nahari, L. W. Cerem, G. Neufeld, and B.-Z. Levi, "Interleukin 6 induces the expression of vascular endothelial growth factor," Journal of Biological Chemistry, vol. 271, no. 2, pp. 736-741, 1996.

[51] B. Dankbar, T. Padró, R. Leo et al., "Vascular endothelial growth factor and interleukin-6 in paracrine tumor-stromal cell interactions in multiple myeloma," Blood, vol. 95, no. 8, pp. 2630-2636, 2000.

[52] K. Kim, S.-Y. Kong, M. Fulciniti et al., "Blockade of the MEK/ ERK signalling cascade by AS703026, a novel selective MEK1/ 2 inhibitor, induces pleiotropic anti-myeloma activity in vitro and in vivo," British Journal of Haematology, vol. 149, no. 4, pp. 537-549, 2010.

[53] D. Puthier, R. Bataille, and M. Amiot, "IL-6 up-regulates mcl1 in human myeloma cells through JAK/STAT rather than ras/ MAP kinase pathway," European Journal of Immunology, vol. 29, no. 12, pp. 3945-3950, 1999.

[54] V. Ramakrishnan and S. Kumar, "PI3K/AKT/mTOR pathway in multiple myeloma: from basic biology to clinical promise," Leukemia \& Lymphoma, vol. 59, no. 11, pp. 2524-2534, 2018.

[55] J. Minarik, Z. Hermanova, P. Petrova, J. Hrbek, J. Krhovska et al., "Prospective study of signalling pathways in myeloma bone disease with regard to activity of the disease, extent of skeletal involvement and correlation to bone turnover markers," European Journal of Haematology, vol. 97, no. 2, pp. 201-207, 2016.

[56] B. D. Hopkins, M. D. Goncalves, and L. C. Cantley, "Obesity and cancer mechanisms: cancer metabolism," Journal of Clinical Oncology, vol. 34, no. 35, pp. 4277-4283, 2016.

[57] M. Pollak, "Insulin, insulin-like growth factors and neoplasia," Best Practice \& Research Clinical Endocrinology \& Metabolism, vol. 22, no. 4, pp. 625-638, 2008.

[58] F. Osório-Costa, G. Z. Rocha, M. M. Dias, and J. B. C. Carvalheira, "Epidemiological and molecular mechanisms aspects linking obesity and cancer," Arquivos Brasileiros de Endocrinologia \& Metabologia, vol. 53, no. 2, pp. 213-226, 2009.

[59] N. van Nieuwenhuijzen, I. Spaan, R. Raymakers, and V. Peperzak, "From MGUS to multiple myeloma, a paradigm for clonal evolution of premalignant cells," Cancer Research, vol. 78, no. 10, pp. 2449-2456, 2018.

[60] C. Pawlyn and G. J. Morgan, "Evolutionary biology of highrisk multiple myeloma," Nature Reviews Cancer, vol. 17, no. 9, pp. 543-556, 2017.

[61] Diabetes Prevention Program Outcomes Study Research Group, T. J. Orchard, M. Temprosa et al., "Long-term effects of the diabetes prevention program interventions on cardiovascular risk factors: a report from the DPP outcomes study," Diabetic Medicine, vol. 30, no. 1, pp. 46-55, 2013.

[62] K. U. A. Adekola, S. Dalva Aydemir, S. Ma, Z. Zhou, S. T. Rosen, and M. Shanmugam, "Investigating and targeting chronic lymphocytic leukemia metabolism with the human immunodeficiency virus protease inhibitor ritonavir and metformin," Leukemia \& Lymphoma, vol. 56, no. 2, pp. 450-459, 2015.

[63] A. E. Quesada, N. D. Nguyen, A. Rios, and R. E. Brown, "Morphoproteomics identifies constitutive activation of the mTORC2/Akt and NF-kappaB pathways and expressions of IGF-1R, Sirt1, COX-2, and FASN in peripheral T-cell lymphomas: pathogenetic implications and therapeutic options," International Journal of Clinical and Experimental Pathology, vol. 7, no. 12, pp. 8732-8739, 2014.

[64] W.-Y. Shi, D. Xiao, L. Wang et al., "Therapeutic metformin/ AMPK activation blocked lymphoma cell growth via inhibition of mTOR pathway and induction of autophagy," Cell Death \& Disease, vol. 3, no. 3, p. e275, 2012.

[65] Y. X. Koo, D. S. Tan, I. B. Tan et al., "Effect of concomitant statin, metformin, or aspirin on rituximab treatment for diffuse large B-cell lymphoma," Leukemia \& Lymphoma, vol. 52, no. 8, pp. 1509-1516, 2011.

[66] F.-M. Zi, J.-S. He, Y. Li et al., "Metformin displays antimyeloma activity and synergistic effect with dexamethasone in in vitro and in vivo xenograft models," Cancer Letters, vol. 356, no. 2, pp. 443-453, 2015.

[67] S. Jagannathan, M. A. Y. Abdel-Malek, E. Malek et al., "Pharmacologic screens reveal metformin that suppresses GRP78-dependent autophagy to enhance the anti-myeloma effect of bortezomib," Leukemia, vol. 29, no. 11, pp. 2184-2191, 2015.

[68] W. Wu, K. Merriman, A. Nabaah et al., "The association of diabetes and anti-diabetic medications with clinical outcomes in multiple myeloma," British Journal of Cancer, vol. 111, no. 3, pp. 628-636, 2014.

[69] Y. Kawano, M. Moschetta, S. Manier et al., "Targeting the bone marrow microenvironment in multiple myeloma," Immunological Reviews, vol. 263, no. 1, pp. 160-172, 2015.

[70] A. K. Mishra and D. Dingli, "Metformin inhibits IL-6 signaling by decreasing IL-6R expression on multiple myeloma cells," Leukemia, vol. 33, no. 11, pp. 2695-2709, 2019.

[71] S. Verdura, E. Cuyas, B. Martin-Castillo, and J. A. Menendez, "Metformin as an archetype immuno-metabolic adjuvant for cancer immunotherapy," Oncoimmunology, vol. 8, no. 10, Article ID e1633235, 2019.

[72] Y.-S. Lee, Y.-S. Kim, S.-Y. Lee et al., "AMP kinase acts as a negative regulator of RANKL in the differentiation of osteoclasts," Bone, vol. 47, no. 5, pp. 926-937, 2010.

[73] Q.-G. Mai, Z.-M. Zhang, S. Xu et al., "Metformin stimulates osteoprotegerin and reduces RANKL expression in osteoblasts and ovariectomized rats," Journal of Cellular Biochemistry, vol. 112, no. 10, pp. 2902-2909, 2011.

[74] J. M. M. Evans, L. A. Donnelly, A. M. Emslie-Smith, D. R. Alessi, and A. D. Morris, "Metformin and reduced risk of cancer in diabetic patients," BMJ, vol. 330, no. 7503, pp. 1304-1305, 2005.

[75] Z.-J. Zhang and S. Li, “The prognostic value of metformin for cancer patients with concurrent diabetes: a systematic review and meta-analysis," Diabetes, Obesity and Metabolism, vol. 16, no. 8, pp. 707-710, 2014.

[76] H. Noto, A. Goto, T. Tsujimoto, and M. Noda, "Cancer risk in diabetic patients treated with metformin: a systematic review and meta-analysis," PLoS One, vol. 7, no. 3, Article ID e33411, 2012.

[77] M. Yin, J. Zhou, E. J. Gorak, and F. Quddus, "Metformin is associated with survival benefit in cancer patients with concurrent type 2 diabetes: a systematic review and metaanalysis," The Oncologist, vol. 18, no. 12, pp. 1248-1255, 2013. 
[78] S. Gandini, M. Puntoni, B. M. Heckman-Stoddard et al., "Metformin and cancer risk and mortality: a systematic review and meta-analysis taking into account biases and confounders," Cancer Prevention Research, vol. 7, no. 9, pp. 867-885, 2014.

[79] R. J. O. Dowling, S. Niraula, V. Stambolic, and P. J. Goodwin, "Metformin in cancer: translational challenges," Journal of Molecular Endocrinology, vol. 48, no. 3, pp. R31-R43, 2012.

[80] M. Pollak, "The insulin and insulin-like growth factor receptor family in neoplasia: an update," Nature Reviews Cancer, vol. 12, no. 3, pp. 159-169, 2012.

[81] M.-Y. El-Mir, V. Nogueira, E. Fontaine, N. Avéret, M. Rigoulet, and X. Leverve, "Dimethylbiguanide inhibits cell respiration via an indirect effect targeted on the respiratory chain complex I," Journal of Biological Chemistry, vol. 275, no. 1, pp. 223-228, 2000.

[82] K. Inoki, T. Zhu, and K.-L. Guan, "TSC2 mediates cellular energy response to control cell growth and survival," Cell, vol. 115, no. 5, pp. 577-590, 2003.

[83] B. Boursi, B. M. Weiss, K. Haynes, R. Mamtani, and Y.-X. Yang, "Reappraisal of risk factors for monoclonal gammopathy of undetermined significance," American Journal of Hematology, vol. 91, no. 6, pp. 581-584, 2016. 